\title{
Investigating the Therapeutic Mechanism of Cannabidiol in a Human Induced Pluripotent Stem Cell (iPSC)-Based Model of Dravet Syndrome
}

\author{
Yishan Sun ${ }^{1,2}$ AND RICARDo E. Dolmetsch ${ }^{1,2}$ \\ ${ }^{1}$ Novartis Institutes for BioMedical Research, Cambridge, Massachusetts 02139, USA \\ ${ }^{2}$ Department of Neurobiology, Stanford University School of Medicine, Stanford, California 94305, USA \\ Correspondence: ricardo.dolmetsch@novartis.com
}

\begin{abstract}
Dravet syndrome is an infantile epileptic encephalopathy primarily caused by loss-of-function variants of the gene $S C N 1 A$. Standard treatment regimens have very limited efficacy to combat the life-threatening seizures in Dravet syndrome or the behavioral-cognitive comorbidities of the disease. Recently there has been encouraging progress in developing new treatments for this disorder. One of the clinical advances is cannabidiol (CBD), a compound naturally found in cannabis and shown to further reduce convulsive seizures in patients when used together with existing drug regimens. Like many other natural products, the exact therapeutic mechanism of CBD remains undefined. Previously we have established a human cellular model of Dravet syndrome by differentiating patient-derived induced pluripotent stem cells (iPSCs) into telencephalic inhibitory and excitatory neurons. Here we have applied this model to investigate the antiepileptic mechanism(s) of CBD at the cellular level. We first determined the effect of escalating the concentrations of CBD on neuronal excitability, using primary culture of rat cortical neurons. We found modulatory effects on excitability at submicromolar concentrations and toxic effects at high concentrations $(15 \mu \mathrm{M})$. We then tested CBD at $50 \mathrm{nM}$, a concentration that corresponds to the estimated human clinical exposure, in telencephalic neurons derived from a patient iPSC line and control cell line H9. This $50 \mathrm{nM}$ of CBD increased the excitability of inhibitory neurons but decreased the excitability of excitatory neurons, without changing the amplitude of sodium currents in either cell type. Our findings suggest a cell type-dependent mechanism for the therapeutic action of CBD in Dravet syndrome that is independent of sodium channel activity.
\end{abstract}

\section{INTRODUCTION}

Dravet syndrome, a catastrophic form of pediatric epilepsy, is characterized by seizures appearing early in infancy and progressing throughout childhood (Dravet et al. 2011). Developmental comorbidities affecting sensory, motor, autonomic, and communicative functions are also hallmarks of Dravet syndrome and contribute significantly to the disease burden (Villas et al. 2017). More than $70 \%$ of Dravet syndrome patients are affected by de novo haploinsufficiency (heterozygous loss-of-function) mutations in the SCN1A gene (Catterall et al. 2010; Escayg and Goldin 2010). SCN1A encodes $\mathrm{Na}_{\mathrm{v}} 1.1$, a voltage-gated sodium channel showing preferential expression in inhibitory interneurons in the brain (Yu et al. 2006; Ogiwara et al. 2007). Upon firing action potentials, the inhibitory interneurons release the inhibitory neurotransmitter GABA to dampen excessive activity of excitatory neurons and orchestrate rhythmic activity in neural circuits (Kepecs and Fishell 2014). In mouse models of Dravet syndrome, $\mathrm{Na}_{\mathrm{v}} 1.1$ haploinsufficiency leads to compromised interneuron function, hypersynchrony in neural circuits, and epileptic seizures. The mouse models have also shed light on a number of ensuing pathophysiological effects on the developing brain, which include compensatory regulation of interneuron activity (Favero et al. 2018), maladaptation of excitatory neurons (Mistry et al. 2014), and neuroinflammatory responses involving glial and vascular cell types (Hawkins et al. 2019). Notably, the primary deficit in interneuron function can persist into adulthood in patients, as revealed by a transcranial magnetic stimulation (TMS) assessment of the neuronal network of the motor cortex during adulthood (Stern et al. 2017).

Using a method to generate telencephalic interneurons and excitatory neurons from patient-derived iPSCs, we have previously established a human cellular model of Dravet syndrome (Sun et al. 2016). ${ }^{3}$ We observed reduction in sodium current density and action potential output in the interneurons generated from patient iPSCs, in comparison to those from control iPSCs as well as a reference embryonic stem cell line H9. In contrast, the excitatory neurons did not show phenotypic differences between patients and controls. Reminiscent of the expression profile in mice, $\mathrm{Na}_{\mathrm{v}} 1.1$ mRNA was expressed at higher levels in

\footnotetext{
${ }^{3}$ Two other iPSC-based studies that characterized inhibitory interneurons alone also revealed functional deficits of the interneurons (Higurashi et al. 2013; Liu et al. 2016). However, another two studies have showed different results - one of the two showed increased excitability of patient-derived excitatory neurons (Jiao et al. 2013), and the other reported increased excitability of both excitatory neurons and inhibitory neurons derived from patients (Liu et al. 2013).

(C) 2018 Sun and Dolmetsch. This article is distributed under the terms of the Creative Commons Attribution-NonCommercial License, which permits reuse and redistribution, except for commercial purposes, provided that the original author and source are credited.
} 
the interneurons than the excitatory neurons in our human cellular model. Furthermore, shRNA-mediated $\mathrm{Na}_{\mathrm{v}} 1.1$ knockdown in interneurons derived from healthy subjects phenocopied patient-derived interneurons, whereas deficits in patient-derived interneurons were rescued by a lentivirus vector expressing wild-type $\mathrm{Na}_{\mathrm{v}} 1.1$ (Sun et al. 2016).

The finding of cell type-specific phenotypes from patient-derived neurons led us to test the effects of therapeutic compounds on these cells. We focused on cannabidiol (CBD), an effective adjunct therapy that reduces convulsive seizures when combined with the current standard of care in patients (Devinsky et al. 2017). The precise antiepileptic mechanism of CBD has not been established, and the corresponding pharmacological potency remains undefined. Through an in vitro dose range finding in rat cortical neuronal culture and estimation of brain exposure from human clinical pharmacokinetic data, we identified $50 \mathrm{nM}$ as a therapeutically relevant concentration for testing in human neurons. This $50 \mathrm{nM}$ of CBD enhanced the excitability of patient-derived inhibitory neurons but down-regulated the excitability of patient-derived excitatory neurons. These findings suggest that CBD may act by reciprocally modulating excitatory and inhibitory neurons, thereby rebalancing network excitability in the context of Dravet syndrome.

\section{RESULTS}

To determine a therapeutically relevant concentration of CBD for testing in the iPSC-based disease model, we did a pilot study using 13- to 14-d-old culture of rat cortical neurons. We loaded the rat neurons with a fluorescent dye indicating levels of intracellular calcium and imaged the neurons using the FLIPR Tetra cellular screening system. FLIPR integrates dye signals from all neurons in a field of view and makes a time-lapse assessment of summed neuronal activity from the field. The FLIPR signal was reduced by the application of Nifedipine $(3.3 \mu \mathrm{M})$, a blocker of L-type voltage-gated calcium channels, demonstrating that the assay is sensitive to neuronal activity (Fig. 1). Application of CBD (with a 6-min incubation) led to a concentration-dependent reduction of the FLIPR signal, and the most significant reduction was achieved at $15 \mu \mathrm{M}$, the highest concentration evaluated (Fig. 1). To understand this effect at the single-neuron level, we performed patch clamp recordings from the rat cortical neurons. Surprisingly, $15 \mu \mathrm{M}$ of CBD abolished action potential generation in both the excitatory neurons and inhibitory interneurons (Fig. 2A) while significantly depolarizing the resting membrane potential in both neuronal classes (Fig. 2B). These effects are unlikely to correspond to the therapeutic mechanism of CBD but could arise from toxicity of high concentrations outside the therapeutic window. To further examine if this was the case, we incubated the primary cortical neurons with $15 \mu \mathrm{M}$ of CBD for $48 \mathrm{~h}$. We observed widespread degradation of dendrites and axons, suggesting a toxic effect of the compound at this concentration (Fig. 2C).

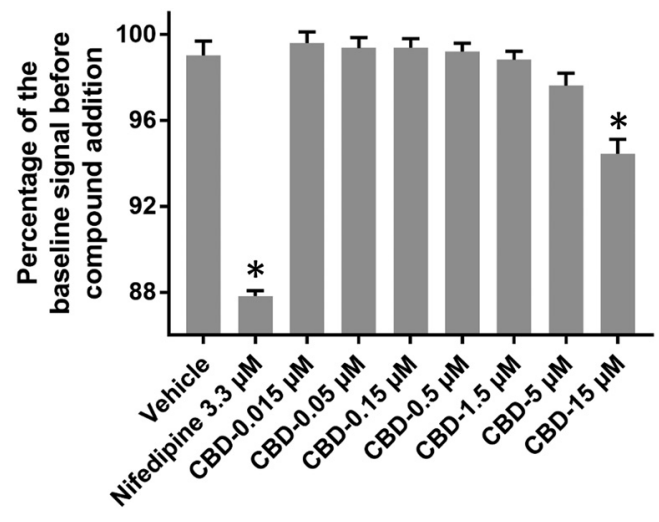

Figure 1. Cannabidiol (CBD) concentration escalation assessed in a neuronal calcium assay using the FLIPR screening system and DIV13 (13 d in vitro) rat cortical neuronal culture. The FLIPR system captures field-level calcium dynamics that reflect the summed activity of all neurons in the imaging field (i.e., the entire assay well). The baseline calcium level was set to $100 \%$. Changes in response to compound additions were normalized to the baseline level and shown on the vertical axis. $N=7$ assay wells per experimental condition. SEM are standard errors of the mean. Asterisk indicates $P<0.05$ by ANOVA and post hoc nonparametric multiple comparisons with the vehicle-treated wells.

Next, we used human pharmacokinetic data to determine therapeutic levels of CBD in the brain. One study reported that an oral dose of $10 \mathrm{mg} / \mathrm{kg} /$ day CBD over $6 \mathrm{wk}$ led to a stabilized mean plasma concentration that ranged between 5.9 and $11.2 \mathrm{ng} / \mathrm{mL}$, which is equivalent to 18.8 to $35.6 \mathrm{nM}$ (Consroe et al. 1991). Another study reported that a single oral dose of $10-15 \mathrm{mg} / \mathrm{kg} \mathrm{CBD}$ with a concomitant intravenous dose of $0.5 \mathrm{mg} / \mathrm{kg}$ fentanyl led to a peak plasma CBD concentration $\left(C_{\max }\right)$ of $221.1 \pm$ $35.6 \mathrm{ng} / \mathrm{mL}$ (i.e., $703.1 \pm 113.2 \mathrm{nM}$ ) (Manini et al. 2015). The efficacious CBD dose in the phase 3 Dravet syndrome clinical trial was $10 \mathrm{mg} / \mathrm{kg} /$ day (Devinsky et al. 2017). Extrapolating the approximately equal distribution of CBD between plasma and brain in rodents (Deiana et al. 2012) to humans, we reasoned that the therapeutically relevant CBD exposure in the brain could be in the submicromolar range. We tested the effects of submicromolar CBD on rat cortical inhibitory interneurons, using a cumulative concentration response regime that applies 5, 50, 500 , and $5 \mu \mathrm{M}$ sequentially and allows $5 \mathrm{~min}$ of preincubation time per concentration. Application of $50 \mathrm{nM}$ and $500 \mathrm{nM}$ of CBD increased the number of action potentials in response to submaximal current injections (i.e., moderate stimulations that do not saturate the output capacity of a neuron when treated with vehicle only), in four out of four rat cortical interneurons examined (Fig. 3). Five micromolar of CBD also enhanced action potential output, but it became less effective than 50 and $500 \mathrm{nM}$ toward the high end of the current injection range (Fig. 3, Neuron \#1, $\# 2$, and \#4). Five nanomolar of CBD increased action potential output in only one of the four neurons examined. Taken together, our results show that CBD increases action potential output of cultured rat cortical interneurons at concentrations that are similar to those observed in clinical studies. 
A

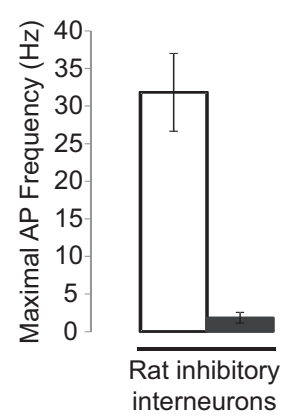

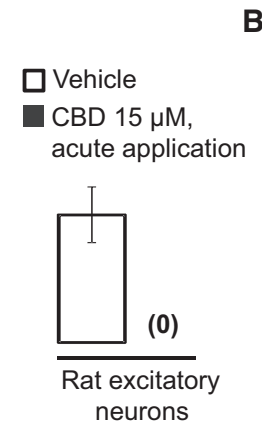

B membrane potential $(\mathrm{mV})$ $\begin{array}{llllll}-65 & -60 & -55 & -50 & -45 & -40\end{array}$
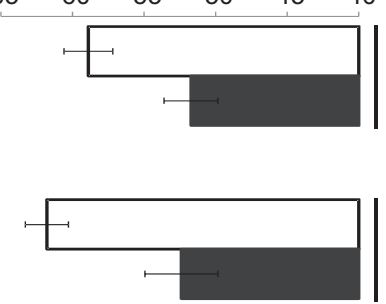

Rat inhibitory interneurons

$\square$ Vehicle

CBD $15 \mu \mathrm{M}$, acute application

Rat excitatory neurons
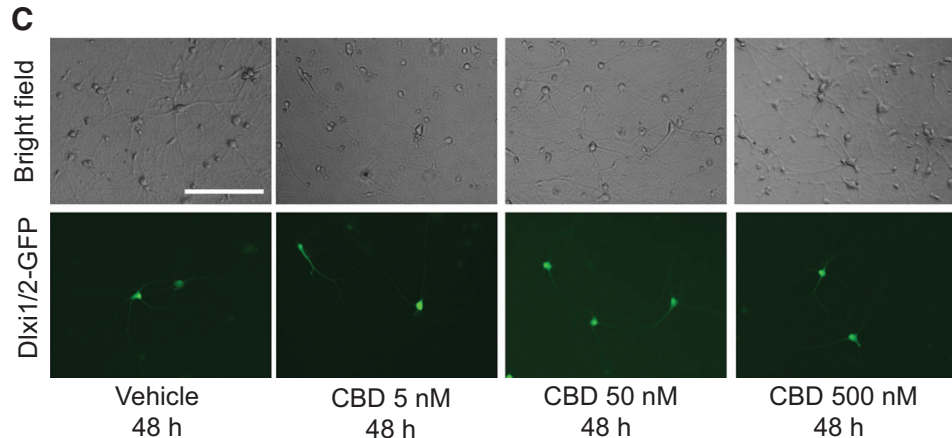

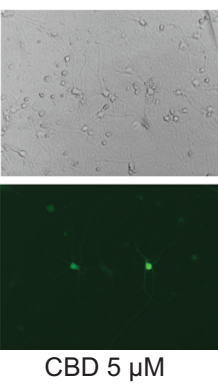

$48 \mathrm{~h}$

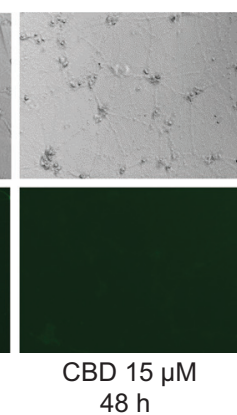

$48 \mathrm{~h}$

Figure 2. Cannabidiol (CBD) at $15 \mu \mathrm{M}$ has detrimental effects on the excitability and viability of DIV13-DIV14 primary culture of rat cortical neurons. (A) $15 \mu \mathrm{M}$ of CBD abolished action potential firing in primary cultures of rat cortical excitatory neurons $(P=0.017$ by $t$-test) and inhibitory neurons $(P=0.019$ by $t$-test). The excitatory neurons were identified by the pyramidal soma and prominent dendrites. The inhibitory neurons were identified by the expression of the lentiviral reporter Dlxi1/2b-GFP (Chen et al. 2013; Sun et al. 2016) and the multipolar GFP-positive neurites. Neurons were recorded in a stationary bath containing $15 \mu \mathrm{M}$ of CBD, and the time of exposure to CBD ranged between $15 \mathrm{~min}$ and $1 \mathrm{~h}$. $(B) 15 \mu \mathrm{M}$ of CBD significantly depolarized the resting membrane potential in both excitatory neurons $(P=0.01$ by $t$-test $)$ and inhibitory neurons $(P=0.002$ by $t$-test $)$ in the culture. $(C)$ Incubating the rat cortical neuronal culture with $15 \mu \mathrm{M}$ of CBD over $48 \mathrm{~h}$ lead to massive cell death. Lower concentrations of CBD up to $5 \mu \mathrm{M}$ were tolerated by the rat neurons within $48 \mathrm{~h}$. Scale bar, 200 microns.

To verify the results in a human neurobiological context directly, we investigated functional effects of CBD in iPSC-derived neurons from patients with Dravet syndrome. We treated inhibitory interneurons derived from a patient iPSC line (6358-2) with $50 \mathrm{nM}$ of CBD and found enhanced action potential output (Fig. 4A). Interneurons derived from the reference $\mathrm{hESC}$ line $\mathrm{H} 9$ responded similarly, especially at submaximal current injections, although the effect was not statistically significant (Fig. 4C). Notably, the amplitude of voltage-dependent sodium currents in the patient-derived interneurons was unchanged, suggesting CBD does not act by directly restoring sodium channel conductance (Table 1; Fig. 4E). We also tested the effects of CBD on excitatory neurons. Fifty nanomolar of CBD suppressed action potential output of both patient-derived and H9-derived excitatory neurons at submaximal levels of current injection (Fig. 4B,D). Once again, no effect was observed on the sodium current amplitude (Table 1; Fig. 4F). Taken together, the results from the iPSC-derived neurons suggest that CBD has opposing effects on neuronal excitability in excitatory and inhibitory human neurons. This effect should favor the restoration of excitation/inhibition balance compromised in Dravet syndrome. Notably the action of CBD was present in both Dravet and control neurons and occurred without increasing neuronal sodium channel conductance.

\section{DISCUSSION}

The iPSC model of Dravet syndrome that we established previously provides an opportunity to evaluate therapeutic compounds, with a goal of translating results from the clinical setting to those at the cellular level, and vice versa. As a proof of principle we studied CBD and found that it modulates neuronal excitability in a cell type-dependent manner that favors rebalancing the activity of excitatory neurons and inhibitory interneurons. Similar results have been reported recently in a Dravet syndrome mouse model, in which CBD positively modulated intrinsic excitability of fast-spiking interneurons in the dentate gyrus but negatively modulated excitability of dentate granule cells (DGCs), while spontaneous GABAergic synaptic transmission onto DGCs were also enhanced by CBD (Kaplan et al. 2017). The authors attributed these effects primarily to the antagonism of CBD to the GPR55 receptor, as a GPR55 receptor antagonist CID16020046 could both mimic and occlude the $\mathrm{CBD}$ effects. It would be interesting to ask if CBD also acts through GPR55 in our iPSC-based cellular model. Of note, in a mouse brain single-cell RNA-seq study, GPR55 was expressed sporadically in very few interneurons and pyramidal cells from the hippocampal CA1 region and the somatosensory cortex (Zeisel et al. 2015). It remains to be elucidated how GPR55 functions in individual neu- 

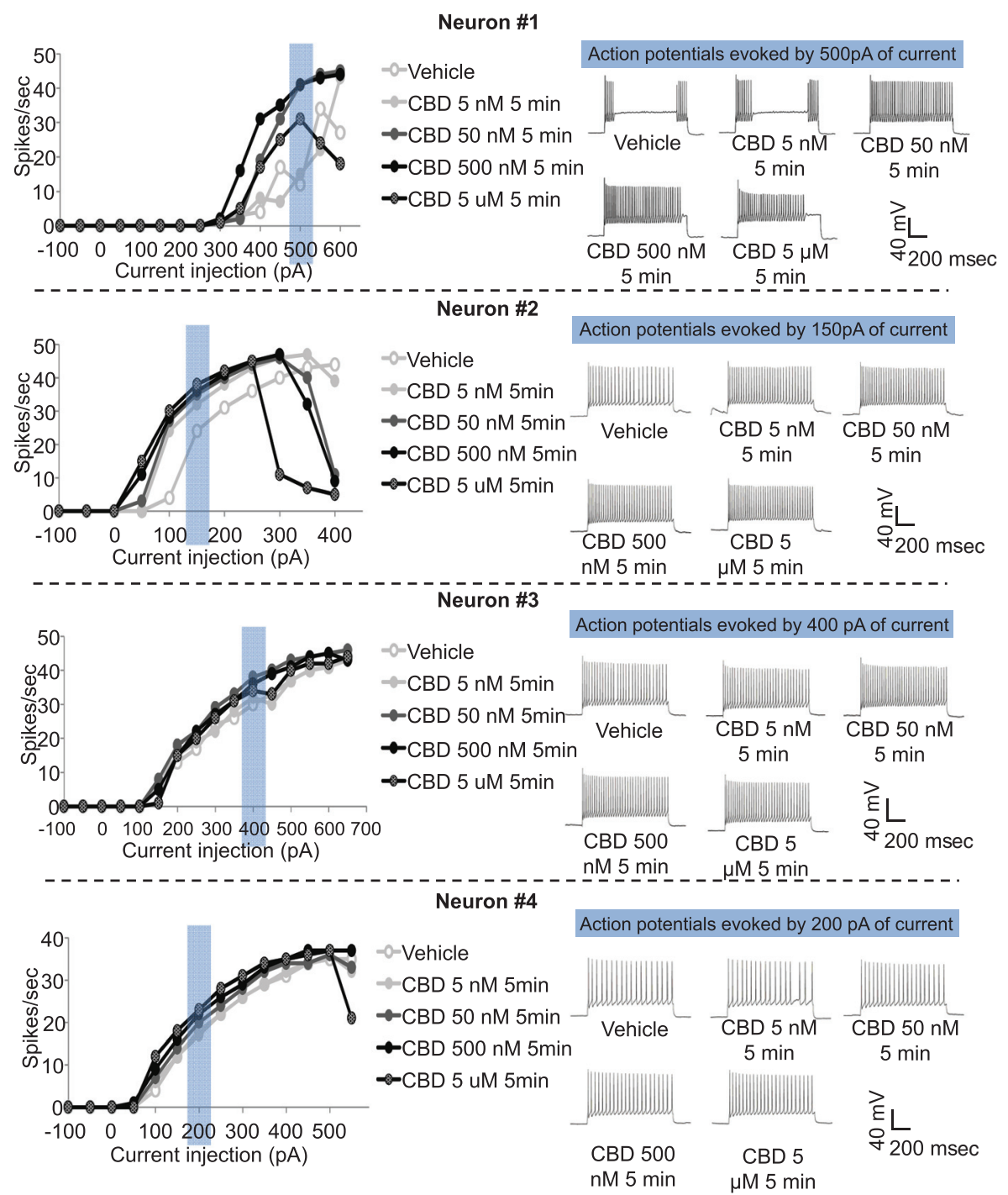

Figure 3. Testing the effect of cannabidiol (CBD) on the excitability of DIV13-DIV14 rat cortical interneurons. Results from four representative neurons are shown. Each neuron was perfused with a cumulative concentration series of CBD from $5 \mathrm{nM}$ to $5 \mu \mathrm{M}$, incubating for $5 \mathrm{~min}$ each. The plots on the left show the input-output relationship for evoked action potentials in each neuron. The inputs are different amounts of current injection (the horizontal axis), and the outputs are the number of evoked action potentials (the vertical axis) at each input level. The traces on the right illustrate the action potentials evoked by a specific submaximal input level as marked in blue on input-output plots, over the CBD concentration series.

rons to tune properties of neural circuits, in both normal and pathophysiological settings. In addition, the testing of CBD in the mouse disease model involved the administering $\mathrm{CBD}$ at $16 \mu \mathrm{M}$ to hippocampal slices, which corresponds to the brain exposure $\left(C_{\max }\right)$ achieved by a 100 $\mathrm{mg} / \mathrm{kg}$ dose of CBD delivered by intraperitoneal injection in rodents (Deiana et al. 2012). The efficacious concentration $(16 \mu \mathrm{M})$ illustrated in the mouse hippocampal slice experiments appears to be in conflict with the toxic concentration $(15 \mu \mathrm{M})$ that we observed in cultured rat cortical neurons. However, there are some important technical differences between the studies. First, a monolayer neuronal culture is directly exposed to a compound in the extracellular solution, whereas in a hippocampal slice neuronal soma and neurites were shielded by surrounding tissue, and the compound exposure is difficult to determine. Second, a compound is likely to bind proteins and lipids in the brain tissue in a slice-based experiment, reducing the free fraction of the compound, so the direct exposure to $\mathrm{CBD}$ is likely to be lower than in the slicebased study.

The current literature also suggests several other possible mechanisms of action for CBD. These include regulation of internal calcium stores (effects shown at $1 \mu \mathrm{M}$ ) (Ryan et al. 2009), interaction with endo-cannabinoid signaling (effects shown at $1 \mu \mathrm{M}$ ) (Ryan et al. 2007), inhibition of voltage-gated sodium channels (with IC50s between 1.9 and $3.8 \mu \mathrm{M}$ among sodium channel subtypes) (Ghovanloo et al. 2018), and selective modulation of resurgent sodium currents of $\mathrm{Na}_{\mathrm{v}} 1.6$ (effects shown at 
A
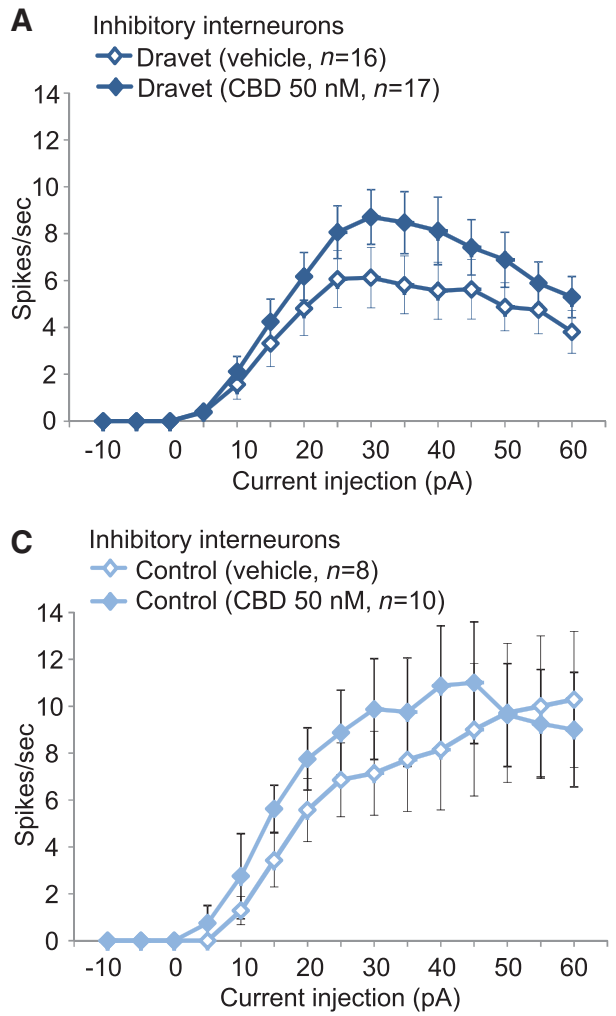

E Inhibitory interneurons

$\leadsto$ Dravet (vehicle, $n=7$ )

$\sim$ Dravet (CBD $50 \mathrm{nM}, n=7$ )

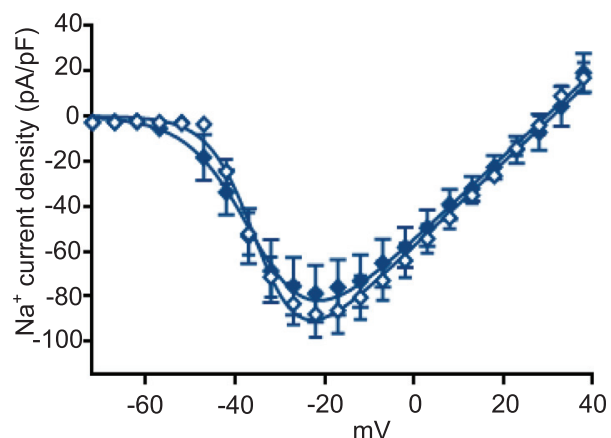

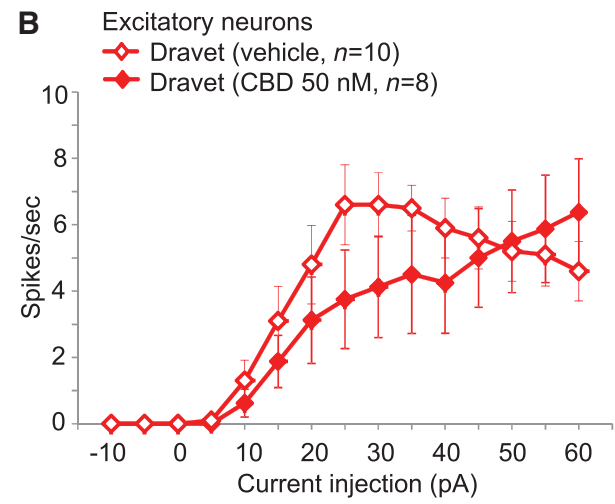

D Excitatory neurons

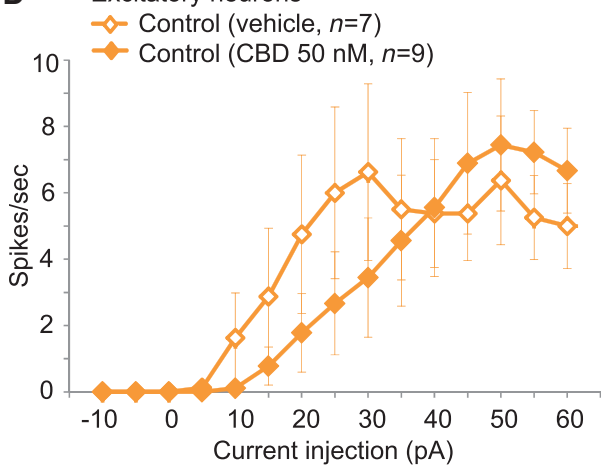

F Excitatory neurons

$\leadsto$ Dravet (vehicle, $n=7$ )

$\rightarrow$ Dravet (CBD $50 \mathrm{nM}, n=5$ )

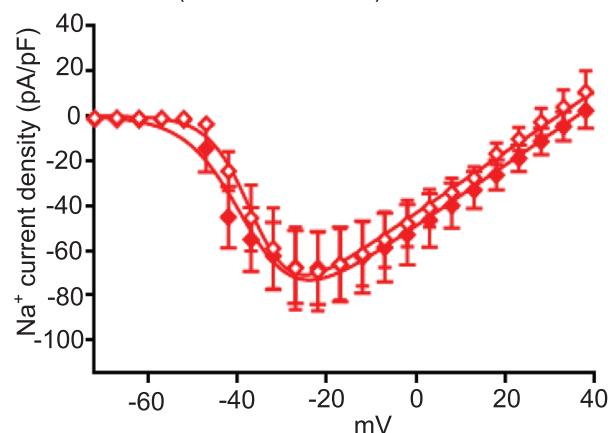

Figure 4. Cannabidiol (CBD) at $50 \mathrm{nM}$ modulates neuronal excitability in a cell type-dependent manner. For data panels $A$ through $D$, two-way ANOVA was performed to evaluate the effect of CBD treatment (factor 1) over a range of current injections (factor 2), and $P$ values are indicated for the effect of CBD treatment. Evoked action potentials are quantified in input-output relationship plots, as defined in Figure 3. Error bars are standard errors of the mean. $(A) 50 \mathrm{nM}$ of CBD moderately enhanced action potential firing in inhibitory neurons derived from Dravet iPSC line 6358-2. $P=0.0002$ for the full current injection range, and $P=0.003$ for the subrange between 10 and $40 \mathrm{pA}$. (B) $50 \mathrm{nM}$ of CBD suppressed firing in excitatory neurons derived from Dravet iPSC line 6358-2, primarily at intermediate current injection levels $(10-40 \mathrm{pA}) . P=0.06$ for the full current range, and $P=0.0039$ for the subrange between 10 and $40 \mathrm{pA}$. (C) Effects of $50 \mathrm{nM}$ of CBD on action potential firing in control inhibitory neurons derived from hESC line H9. $P=$ 0.327 for the full current range, and $P=0.056$ for currents ranged between 10 and $40 \mathrm{pA}$. $(D)$ Effects of $50 \mathrm{nM}$ of CBD on action potential firing in control excitatory neurons derived from hESC line H9. $P=0.035$ for the full current range, and $P=0.04$ for currents ranged between 10 and $40 \mathrm{pA}$. $(E, F) 50 \mathrm{nM}$ of CBD did not alter the voltage-dependent activation of $\mathrm{Na}^{+}$currents $\left(I_{\mathrm{Na}}\right)$ in the Dravet inhibitory neurons $(E)$ or excitatory neurons $(F)$.

$1 \mu \mathrm{M})$ (Patel et al. 2016). Further investigation of all of these possibilities, or unbiased target identification screens, may better define the antiepileptic mechanism of CBD versus adverse effects of the molecule and inform the discovery of new medicines based on this unique natural product.

\section{MATERIAL AND METHODS \\ Rat Cortical Neuronal Culture}

The cerebral cortices of E18 Sprague-Dawley rat embryos were isolated, dissociated into single cells with papain, and cultured at the density of 125,000 cells $/ \mathrm{cm}^{2}$. In 
Table 1. Neuronal sodium channel activation in the presence or absence of $50 \mathrm{nM}$ of cannabidiol (CBD) evaluated in Dravet iPSCderived neurons (cell line: Dravet-iPSC-6358-2)

\begin{tabular}{|c|c|c|c|c|}
\hline \multirow{2}{*}{$\begin{array}{l}\text { Parameter values: } \\
\text { mean }(95 \% \mathrm{CI})\end{array}$} & \multicolumn{2}{|c|}{ Excitatory neurons } & \multicolumn{2}{|c|}{ Inhibitory neurons } \\
\hline & Vehicle (seven cells) & $\mathrm{CBD}$ at $50 \mathrm{nM}$ (five cells) & Vehicle (seven cells) & $\mathrm{CBD}$ at $50 \mathrm{nM}$ (seven cells) \\
\hline$I_{\max }(\mathrm{pA} / \mathrm{pF})$ & $73.61(28.93,118.3)$ & $70.12^{\mathrm{p} 1}(23.21,117.0)$ & $89.32(64.85,113.8)$ & $82.63^{\mathrm{p} 4}(50.66,114.6)$ \\
\hline$V_{\mathrm{R}}(\mathrm{mV})$ & $35.16(26.88,43.44)$ & $39.12^{\mathrm{p} 2}(25.62,52.62)$ & $31.56(28.34,34.78)$ & $34.00^{\mathrm{p} 5}(25.04,42.95)$ \\
\hline$V_{1 / 2}(\mathrm{mV})$ & $-34.19(-38.94,-29.44)$ & $-36.63^{\mathrm{p} 3}(-41.74,-31.53)$ & $-34.19(-36.45,-31.92)$ & $-34.59^{\mathrm{p} 6}(-40.53,-28.64)$ \\
\hline
\end{tabular}

$I_{\max }$ is the maximal inward current detected in a neuron and normalized to the whole cell capacitance. $V_{\mathrm{R}}$ is the reversal potential for sodium currents. $V_{1 / 2}$ describes the membrane potential at which $50 \%$ of the sodium channels are activated. $V_{\mathrm{R}}$ and $V_{1 / 2}$ were determined for each neuron by curve fitting in GraphPad Prism using the equation $I=g *\left(V-V_{\mathrm{R}}\right) /\left(1+\exp \left[-0.03937 * z *\left(V-V_{1 / 2}\right)\right]\right)$ (see Materials and Methods). $95 \%$ CI is the $95 \%$ confidence interval. $\mathrm{p} 1=0.896, \mathrm{p} 2=0.6154$, and p3 $=0.5149$, in corresponding $t$-tests between vehicle- and CBD-treated excitatory neurons. p4 $=0.6912$, p5 $=0.6251$, and $\mathrm{p} 6$ $=0.9039$, in corresponding $t$-tests between vehicle- and CBD-treated inhibitory neurons.

the experiment described in Figure 2, the culture was transduced with the Dlxi1/2b-GFP lentivirus at DIV4 to label inhibitory interneurons (Sun et al. 2016). The isolation of rat cortical tissue was performed in accordance with lab protocols governed by the animal welfare committee (AWC) of the Novartis Institutes for BioMedical Research and the institutional animal care and use committee (IACUC) of Stanford University.

\section{Neuronal Calcium Assay Using the FLIPR Tetra Cellular Screening System}

Rat cortical neurons were grown in 384-well poly-Dlysine coated plates at a density of 16,000 cells per well. On DIV13, the neurons were loaded with the Calcium 6 dye (Molecular Devices) according to the user instructions. Extra potassium chloride $(\mathrm{KCl})$ was added at the time of dye loading so that the extracellular potassium concentration was increased by $7 \mathrm{mM}$, a condition to promote spontaneous neuronal activity. Compounds for testing were prediluted on a 384-well ligand plate according to the targeted concentrations and volumes of dispensing and mixing. FLIPR Tetra was programmed to sample whole-well Calcium 6 signals at $1 \mathrm{~Hz}$ for $130 \mathrm{sec}$ and then $0.25 \mathrm{~Hz}$ for another $360 \mathrm{sec}$, with compound dispensing occurring immediately after the first $101-\mathrm{Hz}$ reads. Per well, readouts from the first $10 \mathrm{sec}$ were averaged as the baseline, and readouts from the last $100 \mathrm{sec}$ were averaged and normalized as a percentage change relative to the baseline. Data were first analyzed sequentially using the ScreenWorks software (Molecular Devices) and GraphPad Prism.

\section{Human iPSC Generation}

The iPSC line 6358-2 was generated through reprogramming of skin fibroblasts from a patient with Dravet syndrome who is heterozygous for missense mutation p.S1328P in $\mathrm{Na}_{\mathrm{v}} 1.1$. The generation of this cell line and the experiments described in Figure 4 were all conducted at Stanford University according to informed consent and in compliance with research protocols approved by IRB and SCRO of Stanford University. Technical details on the iPSC generation can be found in our previous publication (Sun et al. 2016).

\section{Directed Differentiation of Human ES/iPS Cells into Telencephalic Excitatory Neurons and Inhibitory Interneurons}

The differentiation procedure was performed in four stages. In stage 1, human ES/iPS cell colonies were detached from DR4 MEF feeders grown as suspension culture for $5 \mathrm{~d}$ to form embryoid bodies (EBs). In stage 2, the floating EBs were reattached to a 6-cm culture dish for the induction of neural rosettes over $10 \mathrm{~d}$. At the end of this stage, the neural rosettes were visually identified and isolated by mechanical picking. In stage 3 , neural progenitors in the rosettes were plated for postmitotic neuronal differentiation over $20 \mathrm{~d}$. In stage 4 , nascent neurons were cocultured with rat cortical astrocyte feeders for functional maturation. At stages 1, 2, and 3, diverging patterning factors were added to the cell media to promote the generation of either (a) dorsal telencephalic neural stem cells and progenitors (via stages 1 and 2) and excitatory neurons (via stage 3 ) or (b) ventral telencephalic neural stem cells progenitors (via stages 1 and 2) and inhibitory interneurons (via stage 3). CaMKII-GFP and Dlxi1/2-GFP reporter lentiviruses were introduced during stage 3 to label the differentiating excitatory neurons and inhibitory neurons, respectively. Medium recipes and other technical details can be found in our previous publication (Sun et al. 2016).

\section{Patch Clamp Electrophysiology}

Patch clamp recordings were performed with identical instrument configuration and solution recipes as described previously (Sun et al. 2016). Data in Table 1 were based on curve fitting with equation

$$
I=g^{*}\left(V-V_{\mathrm{R}}\right) /\left(1+\exp \left[-0.03937^{*} z^{*}\left(V-V_{1 / 2}\right)\right]\right),
$$

where $I$ is the amplitude of the current peak, $g$ is a factor reflecting the number of active channels in the cell recorded, $V$ is the membrane potential under which the current develops, $z$ is the apparent gating charge, and $V_{1 / 2}$ is the half-maximal activation potential. Data were initially analyzed with the PATCHMASTER software (HEKA). Further analysis was performed using IgorPRO, Microsoft Excel, and GraphPad Prism. 


\section{Pharmacological Reagents}

Cannabidiol solution was purchased from SigmaAldrich as an exempt preparation. Nifedipine was purchased from Tocris and dissolved in DMSO.

\section{ACKNOWLEDGMENTS}

The authors of this study would like to thank the family who donated skin fibroblasts and consented to their use in iPSC-based research at Stanford University and all previous collaborators at Stanford University and UCSF, as well as colleagues in the Neuroscience department of Novartis who provided scientific advice and technical assistance. Some findings of this work have been presented at the Dravet Syndrome Research Roundtable in 2016. Thanks to the Dravet syndrome research community for their input and support.

\section{REFERENCES}

Catterall WA, Kalume F, Oakley JC. 2010. $\mathrm{Na}_{\mathrm{V}} 1.1$ channels and epilepsy. J Physiol 588: 1849-1859. doi:10.1113/jphysiol .2010 .187484

Chen Y-JJ, Vogt D, Wang Y, Visel A, Silberberg SN, Nicholas CR, Danjo T, Pollack JL, Pennacchio LA, Anderson S, et al. 2013. Use of "MGE enhancers" for labeling and selection of embryonic stem cell-derived medial ganglionic eminence (MGE) progenitors and neurons. PLoS One 8: e61956. doi:10.1371/journal.pone.0061956

Consroe P, Laguna J, Allender J, Snider S, Stern L, Sandyk R, Kennedy K, Schram K. 1991. Controlled clinical trial of cannabidiol in Huntington's disease. Pharmacol Biochem Behav 40: 701-708. doi:10.1016/0091-3057(91)90386-G

Deiana S, Watanabe A, Yamasaki Y, Amada N, Arthur M, Fleming S, Woodcock H, Dorward P, Pigliacampo B, Close S, et al. 2012. Plasma and brain pharmacokinetic profile of cannabidiol (CBD), cannabidivarine (CBDV), $\Delta^{9}$-tetrahydrocannabivarin (THCV) and cannabigerol (CBG) in rats and mice following oral and intraperitoneal administration and CBD action on obsessive-compulsive behaviour. Psychopharmacology (Berl) 219: 859-873. doi:10.1007/s00213-011-2415-0

Devinsky O, Cross JH, Laux L, Marsh E, Miller I, Nabbout R, Scheffer IE, Thiele EA, Wright S. 2017. Trial of cannabidiol for drug-resistant seizures in the Dravet syndrome. $N$ Engl J Med 376: 2011-2020. doi:10.1056/NEJMoa1611618

Dravet C, Bureau M, Dalla Bernardina B, Guerrini R. 2011. Severe myoclonic epilepsy in infancy (Dravet syndrome) 30 years later. Epilepsia 52: 1-2. doi:10.1111/j.1528-1167.2011.02993.x

Escayg A, Goldin AL. 2010. Sodium channel SCN1A and epilepsy: mutations and mechanisms. Epilepsia 51: 1650-1658. doi:10.1111/j.1528-1167.2010.02640.x

Favero M, Sotuyo NP, Lopez E, Kearney JA, Goldberg EM. 2018. A transient developmental window of fast-spiking interneuron dysfunction in a mouse model of Dravet syndrome. $J$ Neurosci 38: 7912-7927. doi:10.1523/JNEUROSCI.0193-18 .2018

Ghovanloo M-R, Shuart NG, Mezeyova J, Dean RA, Ruben PC, Goodchild SJ. 2018. Inhibitory effects of cannabidiol on voltage-dependent sodium currents. J Biol Chem 293: 16546 16558. doi:10.1074/jbc.RA118.004929

Hawkins NA, Calhoun JD, Huffman AM, Kearney JA. 2019. Gene expression profiling in a mouse model of Dravet syndrome. Exp Neurol 311: 247-256. doi:10.1016/j.expneurol .2018 .10 .010

Higurashi N, Uchida T, Lossin C, Misumi Y, Okada Y, Akamatsu W, Imaizumi Y, Zhang B, Nabeshima K, Mori MX, et al. 2013. A human Dravet syndrome model from patient induced pluripotent stem cells. Mol Brain 6: 19. doi:10.1186/17566606-6-19
Jiao J, Yang Y, Shi Y, Chen J, Gao R, Fan Y, Yao H, Liao W, Sun X-F, Gao S. 2013. Modeling Dravet syndrome using induced pluripotent stem cells (iPSCs) and directly converted neurons. Hum Mol Genet 22: 4241-4252. doi:10.1093/hmg/ $\operatorname{ddt} 275$

Kaplan JS, Stella N, Catterall WA, Westenbroek RE. 2017. Cannabidiol attenuates seizures and social deficits in a mouse model of Dravet syndrome. Proc Natl Acad Sci 114: 11229 11234. doi:10.1073/pnas.1711351114

Kepecs A, Fishell G. 2014. Interneuron cell types are fit to function. Nature 505: 318-326. doi:10.1038/nature12983

Liu Y, Lopez-Santiago LF, Yuan Y, Jones JM, Zhang H, O’Malley HA, Patino GA, O’Brien JE, Rusconi R, Gupta A, et al. 2013. Dravet syndrome patient-derived neurons suggest a novel epilepsy mechanism. Ann Neurol 74: 128-139. doi:10 $.1002 /$ ana.23897

Liu J, Gao C, Chen W, Ma W, Li X, Shi Y, Zhang H, Zhang L, Long Y, Xu H, et al. 2016. CRISPR/Cas9 facilitates investigation of neural circuit disease using human iPSCs: mechanism of epilepsy caused by an SCN1A loss-of-function mutation. Transl Psychiatry 6: e703. doi:10.1038/tp.2015.203

Manini AF, Yiannoulos G, Bergamaschi MM, Hernandez S, Olmedo R, Barnes AJ, Winkel G, Sinha R, Jutras-Aswad D, Huestis MA, et al. 2015. Safety and pharmacokinetics of oral cannabidiol when administered concomitantly with intravenous fentanyl in humans. $J$ Addict Med 9: 204-210. doi:10 .1097/ADM.0000000000000118

Mistry AM, Thompson CH, Miller AR, Vanoye CG, George AL, Kearney JA. 2014. Strain- and age-dependent hippocampal neuron sodium currents correlate with epilepsy severity in Dravet syndrome mice. Neurobiol Dis 65: 1-11. doi:10 $.1016 /$ j.nbd.2014.01.006

Ogiwara I, Miyamoto H, Morita N, Atapour N, Mazaki E, Inoue I, Takeuchi T, Itohara S, Yanagawa Y, Obata K, et al. 2007. $\mathrm{Na}_{\mathrm{v}} 1.1$ localizes to axons of parvalbumin-positive inhibitory interneurons: a circuit basis for epileptic seizures in mice carrying an Scnla gene mutation. J Neurosci 27: 5903-5914. doi:10.1523/JNEUROSCI.5270-06.2007

Patel RR, Barbosa C, Brustovetsky T, Brustovetsky N, Cummins TR. 2016. Aberrant epilepsy-associated mutant $\mathrm{Na}_{\mathrm{v}} 1.6$ sodium channel activity can be targeted with cannabidiol. Brain 139: 2164-2181. doi:10.1093/brain/aww129

Ryan D, Drysdale AJ, Pertwee RG, Platt B. 2007. Interactions of cannabidiol with endocannabinoid signalling in hippocampal tissue. Eur J Neurosci 25: 2093-2102. doi:10.1111/j.14609568.2007.05448.x

Ryan D, Drysdale AJ, Lafourcade C, Pertwee RG, Platt B. 2009. Cannabidiol targets mitochondria to regulate intracellular $\mathrm{Ca}^{2+}$ levels. J Neurosci 29: 2053-2063. doi:10.1523/JNEUROSCI .4212-08.2009

Stern WM, Sander JW, Rothwell JC, Sisodiya SM. 2017. Impaired intracortical inhibition demonstrated in vivo in people with Dravet syndrome. Neurology 88: 1659-1665. doi:10 .1212/WNL.0000000000003868

Sun Y, Paşca SP, Portmann T, Goold C, Worringer KA, Guan W, Chan KC, Gai H, Vogt D, Chen Y-JJ, et al. 2016. A deleterious $\mathrm{Na}_{\mathrm{v}} 1.1$ mutation selectively impairs telencephalic inhibitory neurons derived from Dravet Syndrome patients. Elife 5: e13073. doi:10.7554/eLife.13073

Villas N, Meskis MA, Goodliffe S. 2017. Dravet syndrome: characteristics, comorbidities, and caregiver concerns. Epilepsy Behav 74: 81-86. doi:10.1016/j.yebeh.2017.06.031

Yu FH, Mantegazza M, Westenbroek RE, Robbins CA, Kalume F, Burton KA, Spain WJ, McKnight GS, Scheuer T, Catterall WA. 2006. Reduced sodium current in GABAergic interneurons in a mouse model of severe myoclonic epilepsy in infancy. Nat Neurosci 9: 1142-1149. doi:10.1038/nn1754

Zeisel A, Munoz-Manchado AB, Codeluppi S, Lonnerberg P, La Manno G, Jureus A, Marques S, Munguba H, He L, Betsholtz C, et al. 2015. Cell types in the mouse cortex and hippocampus revealed by single-cell RNA-seq. Science 347: 1138-1142. doi:10.1126/science.aaa1934 


\title{
Erratum
}

Cold Spring Harb Symp Quant Biol 83 (2018) doi: 10.1101/sqb.2018.83.038174

\section{Erratum: Investigating the Therapeutic Mechanism of Cannabidiol in a Human Induced Pluripotent Stem Cell (iPSC)-Based Model of Dravet Syndrome}

\author{
Yishan Sun AND Ricardo E. Dolmetsch
}

The title of this article was originally "Investigating the Therapeutic Mechanism of Cannabidiol in a Human Induced Pluripotent Stem Call (iPSC)-Based Cellular Model of Dravet Syndrome." The word "Cell” was misspelled as "Call” as a result of a production error, for which the publisher apologizes. Because "Cell" is redundant with "Cellular," the authors requested that the latter be removed from the title. The title of the current version of the article, shown above, reflects these revisions.

doi: 10.1101/sqb.2018.83.038919 


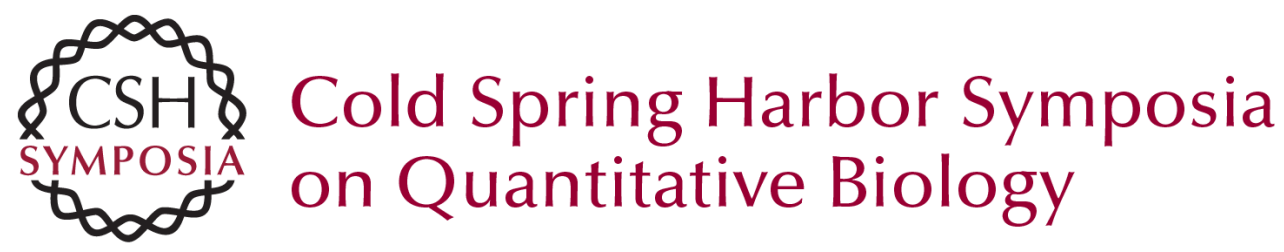

\section{Investigating the Therapeutic Mechanism of Cannabidiol in a Human Induced Pluripotent Stem Cell (iPSC)-Based Model of Dravet Syndrome}

Yishan Sun and Ricardo E. Dolmetsch

Cold Spring Harb Symp Quant Biol 2018 83: 185-191 originally published online June 11, 2019 Access the most recent version at doi:10.1101/sqb.2018.83.038174
Related Content Erratum: Investigating the Therapeutic Mechanism of Cannabidiol in a Human Induced Pluripotent Stem Cell (iPSC)-Based Model of Dravet Syndrome
Yishan Sun and Ricardo E. Dolmetsch
Cold Spring Harb Symp Quant Biol UNKNOWN , 2018 83:
References This article cites 27 articles, 6 of which can be accessed free at: http://symposium.cshlp.org/content/83/185.full.html\#ref-list-1
Articles cited in:
http://symposium.cshlp.org/content/83/185.full.html\#related-urls
Creative This article is distributed under the terms of the
Commons http://creativecommons.org/licenses/by-nc/4.0/, which permits reuse and
License redistribution, except for commercial purposes, provided that the original author and source are credited. Email Alerting
Service $\quad \begin{aligned} & \text { Receive free email alerts when new articles cite this article - sign up in } \\ & \text { the box at the top right corner of the article or click here. }\end{aligned}$ 